\title{
Progressive Symmetrical Erythrokeratoderma-Like Psoriasis: A New Case Report
}

\author{
Waqas S. Abdulwahhab ${ }^{1 *}$, Sumer Baroud ${ }^{2}$, Khaled Adel Al Sayed1 \\ ${ }^{1}$ Department of Dermatology and Venereology, Al Qassimi Hospital, Sharjah, United Arab Emirates \\ ${ }^{2}$ College of Medicine, Sharjah University, Sharjah, United Arab Emirates \\ Email: ^wqs_saad@yahoo.com, sumer.baroud@gmail.com, Khaled_Alsayed13@hotmail.com
}

How to cite this paper: Abdulwahhab, W.S., Baroud, S. and Al Sayed, K.A. (2021) Progressive Symmetrical Erythrokeratoderma-Like Psoriasis: A New Case Report. Journal of Cosmetics, Dermatological Sciences and Applications, 11, 293-303.

https://doi.org/10.4236/jcdsa.2021.114024

Received: August 2, 2021

Accepted: November 29, 2021

Published: December 2, 2021

Copyright $\odot 2021$ by author(s) and Scientific Research Publishing Inc. This work is licensed under the Creative Commons Attribution International License (CC BY 4.0).

http://creativecommons.org/licenses/by/4.0/

\begin{abstract}
Background: Psoriasis, a chronic, systemic, inflammatory disease with prominent skin involvement, affects approximately $2 \%-4 \%$ of the world population. Common variants of psoriasis are plaque psoriasis, inverse psoriasis, guttate psoriasis, erythrodermic psoriasis, pustular form either palmoplantar pustular psoriasis or generalized pustular psoriasis, nail psoriasis, and psoriatic arthritis. Progressive Symmetrical Erythrokeratoderma (PSEK) is a rare genetic disorder, characterized by fixed, well-defined erythematous and hyperkeratotic plaques distributed predominantly on the elbows, knees, trunk, and dorsal surfaces of hands and feet. Clinically has the same presentation to psoriasis especially at early onset and could be confused with psoriasis but histopathological findings and progression of the psoriatic disease can differentiate between both conditions. Aim: To document a new variant of a severe, recalcitrant type of psoriasis with a history of recurrent attacks of exacerbations and partial remissions especially in lesions involving lower extremities that are clinically PSEK-like in presentation, but histopathologically consistent with psoriasis. Case report: A 12-year-old childhood male, known case of Down's syndrome, presented to our clinic with a history of severely pruritic skin rashes involving the perioral area, corners of the mouth, bilateral elbows, dorsal hands, scrotum, and both lower extremities for 6 years duration. The rashes gradually progressed with time to form fixed lesions in the last 2 years. $\mathrm{He}$ was received multiple treatment modalities, including topical steroids, topical vitamin D derivatives, and narrowband UVB phototherapy without significant improvement and the lesions became more worsened over time. Conclusion: psoriasis can be presenting with a new variant of a severe, recalcitrant, and difficult to treat type in Down syndrome cases with a history of
\end{abstract}


recurrent attacks of exacerbations and partial remissions especially in lesions involving lower extremities which are clinically PSEK-like in presentation, but histopathologically consistent with psoriasis. However, early diagnosis and strict management are important in controlling the severity of the condition.

\section{Keywords}

Progressive Symmetrical Erythrokeratoderma, Psoriasis, Down Syndrome, Pruritus, Biological Therapy, Hyperpigmentation

\section{Introduction}

Psoriasis, a chronic, systemic inflammatory disease with prominent skin involvement, affects approximately $2 \%-4 \%$ of the world's population [1] [2] and profoundly impairs patients' health-related quality of life [3] [4] [5] [6].

Common presentations include Plaque psoriasis, inverse psoriasis, guttate psoriasis, erythrodermic psoriasis, generalized pustular psoriasis, and palmoplantar pustular psoriasis. Extracutaneous manifestations of psoriasis include nail abnormalities and psoriatic arthritis [7] [8] [9].

Progressive symmetrical erythrokeratoderma (PSEK) is a rare genetic disorder, characterized by fixed, well-defined erythematous and hyperkeratotic plaques distributed predominantly on the elbows, knees, trunk, and dorsal surfaces of hands and feet. It was first described by Darier [10] in 1911 and later renamed by Gottron [11] in 1922. Hence, it is also known as Gottron's syndrome. PSEK is predominantly inherited as an autosomal dominant trait, but there are reports that $40 \%$ of the cases occur sporadically [12]. Onset is usually in infancy with gradual progression of the lesions in childhood and thereafter it tends to become stable. The diagnosis is mainly clinical, and positive family history can be found in few cases.

Darier described PSEK as a rare keratinization disorder, characterized by non-migratory keratotic plaques on an erythematous base, appearing insidiously and slowly on circinate areas, surrounded by a delicate hyperpigmented projection, with symmetric distribution on knees, elbows, hands, and feet. Transmission in PSEK is hereditary and it results in an autosomal dominant pattern, with sporadic cases occurring in up to $50 \%$ of the total diagnosed. Diagnosis is established on a clinical basis and histopathology is most important to exclude the main differential diagnosis, psoriasis. Histopathology findings show hyperkeratosis, moderate acanthosis with a prominent granular layer. The therapy will be directed according to clinical manifestations. In clinically moderated non-malignant cases, the use of retinoids, keratolytic, and even steroids would be an option. In more extensive cutaneous involvement, systemic therapy is indicated, with retinoids being the main choice. The most commonly used drugs are acitretin and 
etretinate in doses varying between 0.5 to $1.0 \mathrm{mg} / \mathrm{kg}$. Phototherapy has shown good results [13].

In the present case report, we are describing a 12-year-old childhood male known case of Down syndrome with a new variant of a severe, resistant type of psoriasis characterized by a history of recurrent attacks of exacerbations and partial remissions especially in lesions involving lower extremities which is clinically PSEK-like in presentation, but histopathologically consistent with psoriasis. The condition is difficult to treat and failure to multiple topical and systemic therapies but improved on biological injection Secukinumab and methotrexate (MTX) treatments. The consent form was taken from the patient's father for the publication of his condition.

\section{Case Report}

A 12-year-old childhood male, a known case of Down's syndrome, presented to our clinic with a history of severely pruritic skin rashes involving the perioral area, corners of the mouth, bilateral elbows, dorsal hands, scrotum, and both lower extremities for 6 years duration. The rashes gradually progressed over time to form fixed lesions in the last 2 years. He was received multiple treatment modalities, including topical steroids, topical vitamin $\mathrm{D}$ derivatives and narrowband UVB phototherapy without significant improvement and lesions became more worsened over time.

On examination, the patient presented with perioral erythema covered by fine dry scales, angular cheilitis, and fixed well-defined erythematous thick keratotic plaques associated with circinate dried scaly borders in geographical distribution around and within plaques involving both elbows, bilateral dorsal hands, scrotum, and bilateral lower extremities surrounded by expanded projections of hyperpigmentation some in whorled configurations especially at lower legs. The plaques were covered by whitish adherent thick scales and the Psoriasis Area and Severity Index (PASI) score at initial presentation was 24.4 (Figures 1-4). There was no family history of the same presentation or joint involvement.

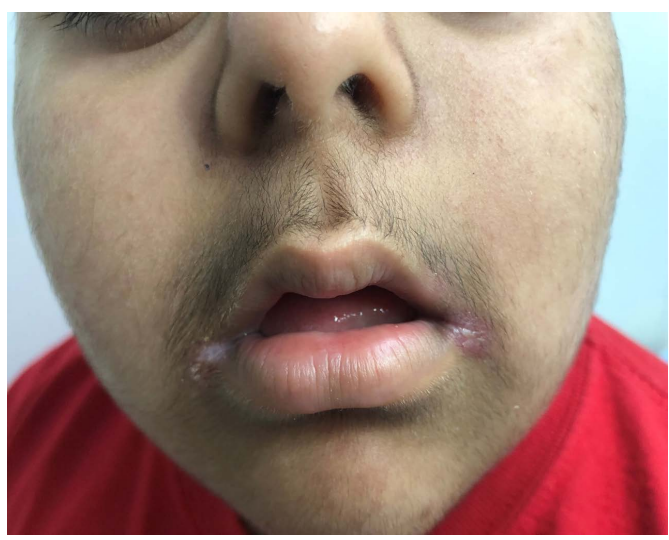

Figure 1. PSEK-like psoriasis showed perioral erythema with mild scales. Note angular cheilitis of a 12-year-old childhood male patient. 


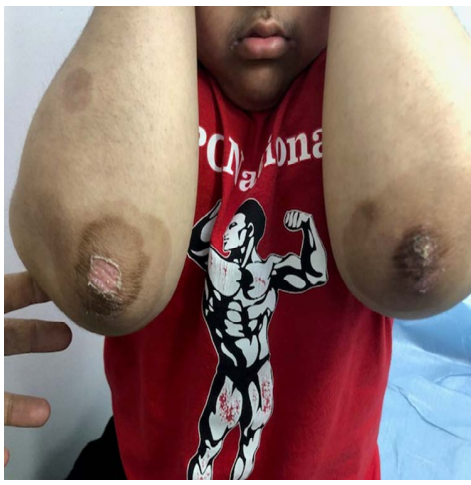

(a)

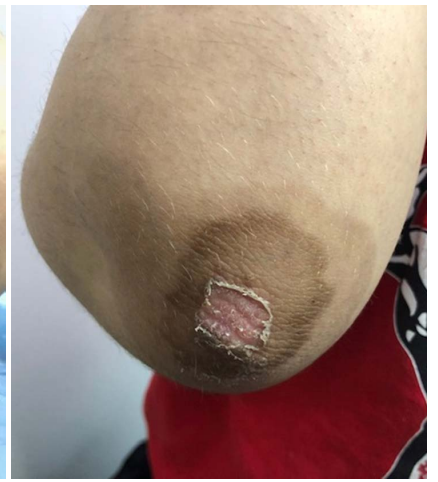

(b)

Figure 2. PSEK-like psoriasis showed well-defined symmetrical hyperkeratotic plaques on dorsal elbows surrounded by expanded projections of hyperpigmentation of a 12-year-old childhood male. Note circinate scales surrounded borders; (a) Both elbows; (b) Right elbow.

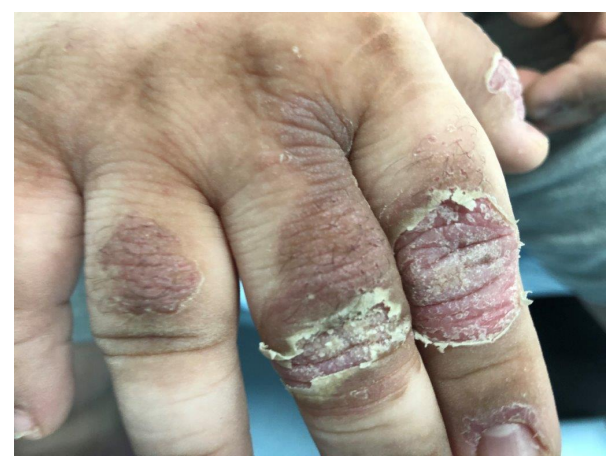

Figure 3. PSEK-like psoriasis showed well-defined erythematous hyperkeratotic plaques covered by adherent whitish scales on the dorsal right hand surrounded by expanded projections of hyperpigmentation of a 12-year-old childhood male. Note circinate whitish scales surrounding borders.

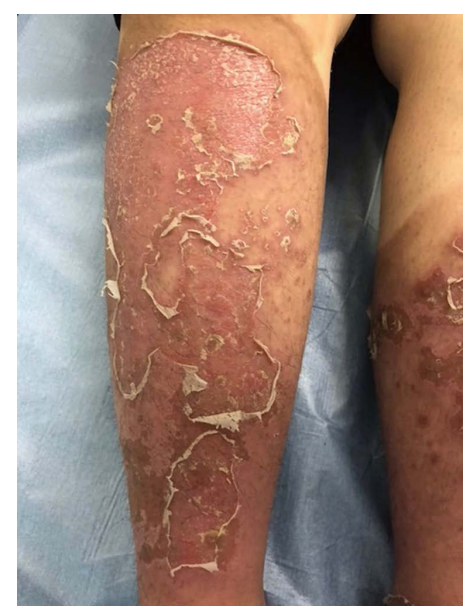

(a)

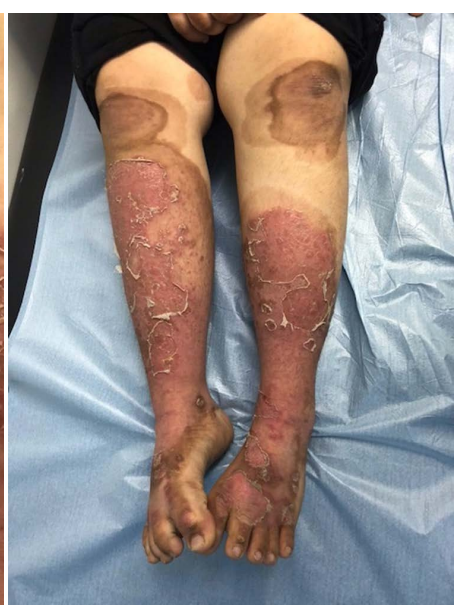

(b)

Figure 4. PSEK-like psoriasis showed well-defined symmetrical hyperkeratotic plaques on bilateral lower legs surrounded by expanded projections of hyperpigmentation of a 12-year-old childhood male. Note geographical distribution of scales around and within lesions. (a) Both lower legs. (b) Right lower leg. 
Routine investigations for patients obtained included CBC, LFT, Lipid profile, electrolyte panel, Iron store, Zinc level, and markers for autoimmune diseases, all of which turned out to be normal. A skin biopsy was taken from the patient for histopathology report and differential diagnoses were included psoriasis, PSEK variabilis sporadic type, pityriasis rubra pilaris, and acrodermatitis enteropathica. The biopsy result was consistent with psoriasis, showing acanthosis, parakeratosis, hypogranulosis with Munro microabscesses in stratum corneum (Figure 5).

Treatment was started with acitretin capsule $25 \mathrm{mg}$ daily for first two months but due to little improved condition stop acitretin capsule and shifted to Adalimumab $40 \mathrm{mg}$ injection every 2 weeks for 2 months then $40 \mathrm{mg}$ weekly for another 2 months after reassessment condition and full investigation for biological therapy to exclude any adverse effects. But due to lack of efficacy and relapsed attacks of exacerbations of pustular psoriasis at lower extremities, stopped adalimumab treatment. Skin swab culture obtained from pustules involved lower legs and result was positive for pseudomonas infection, ciprofloxacin $500 \mathrm{mg}$ BID for 7 days was given. Then Ustekinumab injection, $45 \mathrm{mg}$ subcutaneously was started initially then changed to $90 \mathrm{mg}$ injection for first 3 months then for 2 months and given oral $7.5 \mathrm{mg}$ methotrexate (MTX) tablet weekly. The patient showed partial response in the areas involving the perioral area, elbows, and dorsal hands, however, plaques of the lower legs were resistant to treatment, showed flares of severely pruritic pustular psoriasis and partial remissions with PASI score $=15$ (Figure 6, Figure 7). Patient suffered from repeated vomiting when trying to increase the dose of MTX beyond $7.5 \mathrm{mg}$ dose. Thus stopped Ustekinumab injection, reassess the patient for any side effects or complications with full investigations, and started with Secukinumab injection $150 \mathrm{mg}$ subcutaneously, and continued oral MTX $7.5 \mathrm{mg}$ weekly. 5 weeks after loading therapy, significant improvement in pruritus and lesions of lower legs started to appear where resolved psoriatic plaques leaving mild erythema and expanded areas of post-inflammatory hyperpigmentation accompanied with few remnants fine scales within lesions and PASI score was 4.4 (Figures 8-10). The patient follows

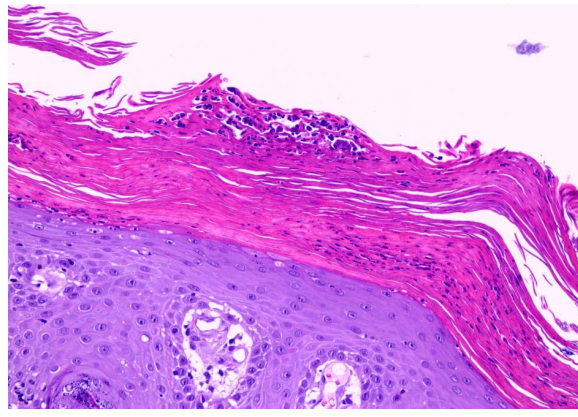

(a)

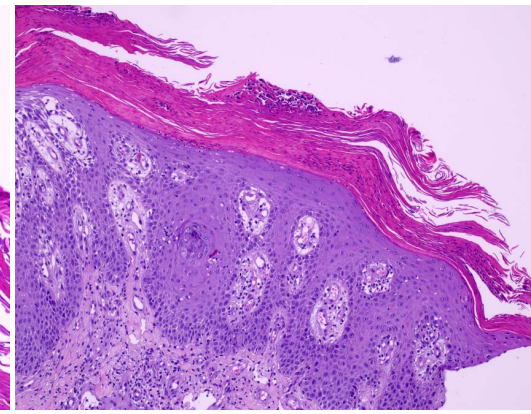

(b)

Figure 5. Hematoxylin and Eosin (H\&E) stained section showed acanthosis, parakeratosis, hypogranulosis, and Munro micro abscess. (a) Original magnification X10; (b) Original magnification X20. 


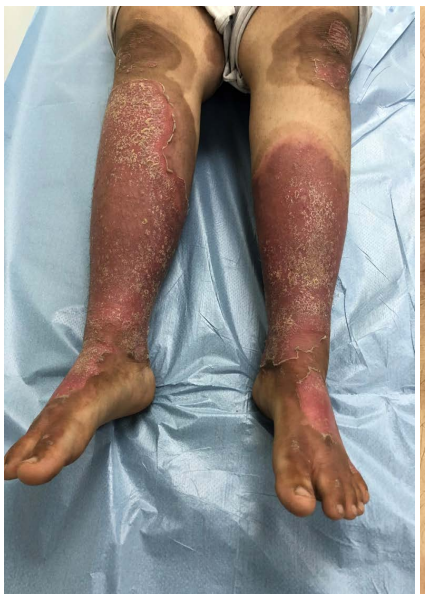

(a)

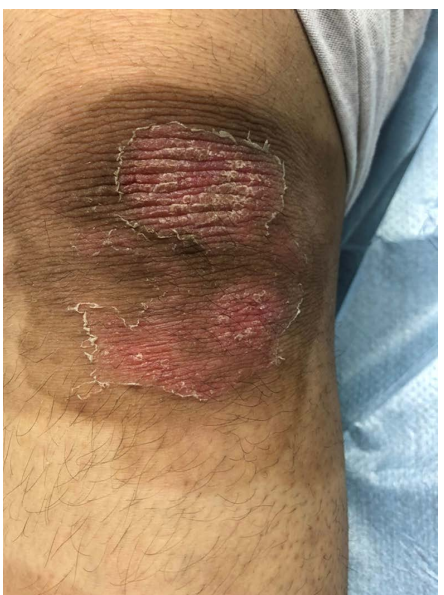

(b)

Figure 6. PSEK-like psoriasis during exacerbation showed well-defined symmetrical hyperkeratotic plaques on lower legs surrounded by expanded projections of hyperpigmentation of a 12-year-old childhood male. Note studded of plaques with tinny pustules indicates pustular psoriasis. (a) Both lower legs; (b) Left knee joint.

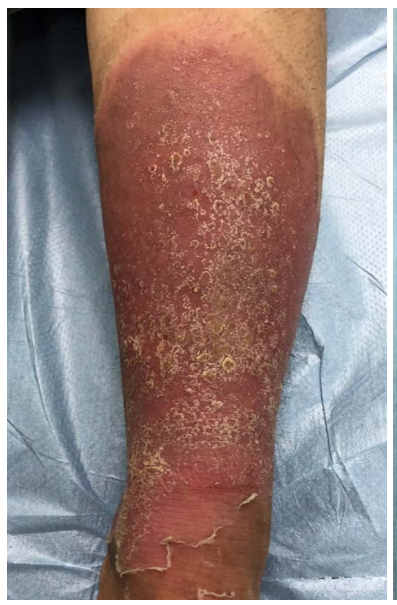

(a)

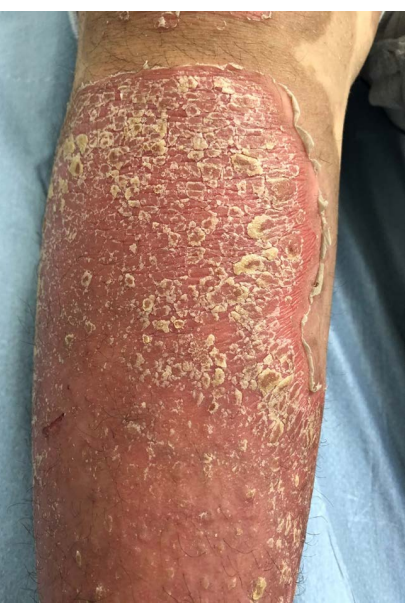

(b)

Figure 7. PSEK-like psoriasis during exacerbation showed well-defined symmetrical hyperkeratotic plaques on lower legs covered by thick adherent whitish scales surrounded by expanded projections of hyperpigmentation of a 12-year-old childhood male. Note studded of plaques with tinny pustules indicate pustular psoriasis. (a) Left lower leg; (b) Right lower leg.

up at 8 weeks later showed continuing response to therapy without signs of relapse (Figure 11). Full assessment for the patient was repeated when changed biological therapy included viral load, QuantiFERON TB Gold test, and CXR to ensure patient safety and exclude any adverse effects.

\section{Discussion}

Approximately $30 \%$ of all patients with plaque psoriasis experience the onset of the disease before 16 years of age [14]. Psoriasis occurs in up to $8 \%$ of patients with Down syndrome and, although poorly characterized, appears to be correlated 


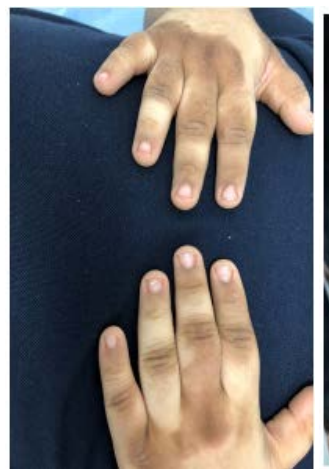

(a)

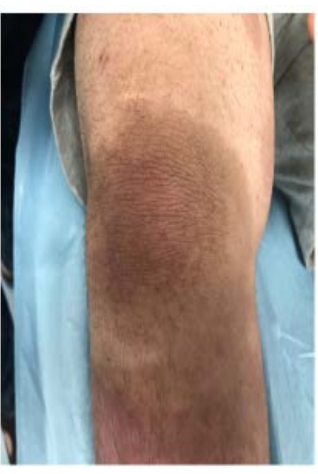

(b)

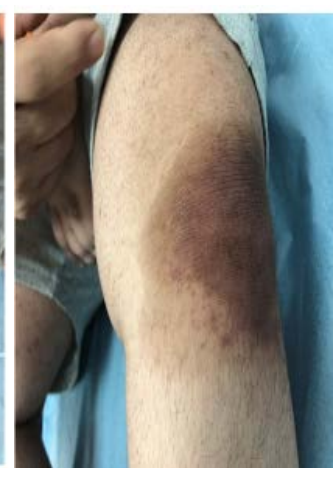

(c)

Figure 8. PSEK-like psoriasis 5 weeks loading treatment with Secukinumab injection and MTX showed complete resolving plaques leaving expanded areas of hyperpigmentation of a 12-year-old childhood male. (a) Bilateral dorsal hands; (b) Right knee joint; (c) Left knee joint.

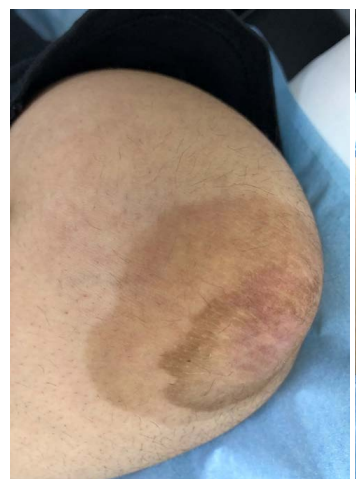

(a)

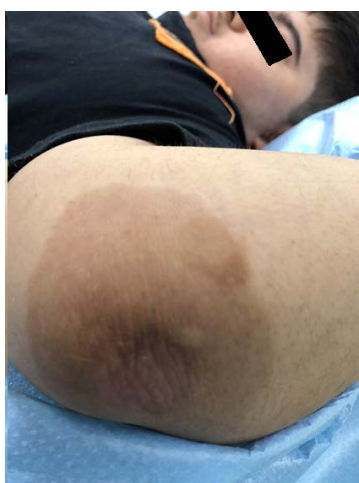

(b)

Figure 9. PSEK-like psoriasis 5 weeks loading treatment with Secukinumab injection and MTX showed complete resolving plaques leaving expanded areas of hyperpigmentation of a 12-year-old childhood male. (a) Right elbow; (b) Left elbow.

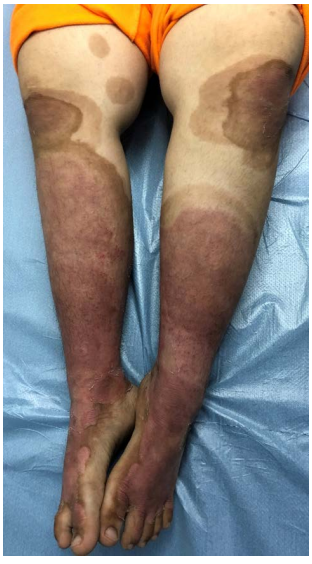

(a)

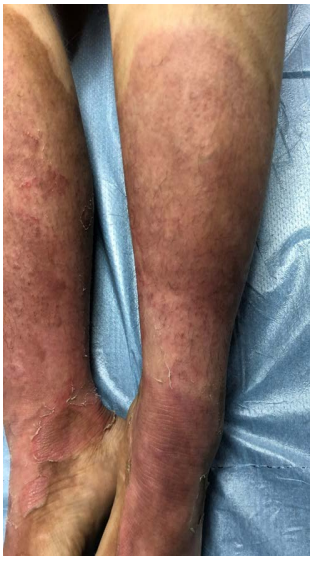

(b)

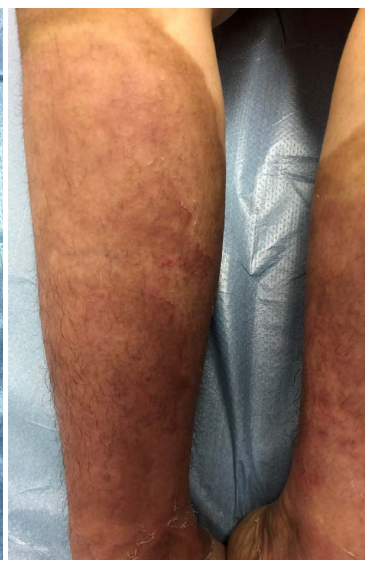

(c)

Figure 10. PSEK-like psoriasis 5 weeks loading treatment with Secukinumab injection and MTX showed resolving plaques leaving mild erythema with scales and expanded areas of hyperpigmentation of a 12-year-old childhood male. (a) Bilateral lower legs; (b) Left lower leg; (c) Right lower leg. 


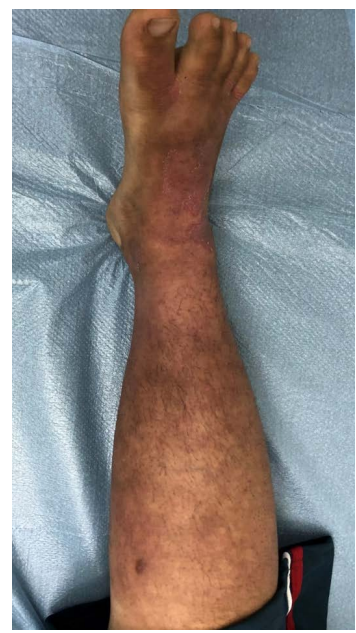

(a)

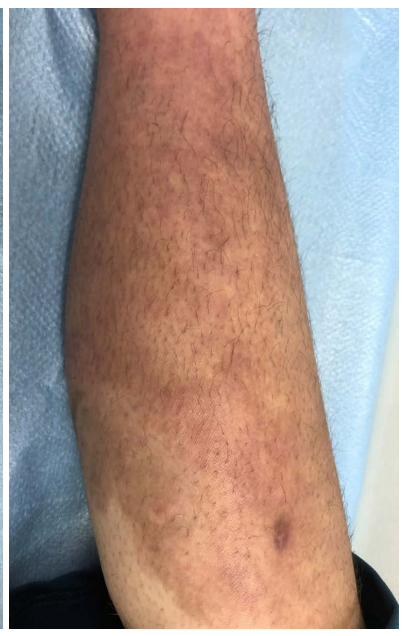

(b)

Figure 11. PSEK-like psoriasis 8 weeks treatment with Secukinumab injection and MTX showed complete resolving plaques leaving slight erythema and expanded areas of hyperpigmentation of a 12-year-old childhood male. (a) Right lower leg; (b) Left lower leg.

with an immune imbalance in cells Th1 and biochemical changes of cyclic nucleotides [15]. The association between plaque psoriasis and Down syndrome is unclear. It was suggested that a dysregulation of the interferon- $\gamma$ system is responsible for susceptibility to plaque psoriasis in the Down syndrome population [16]. Moreover, immunological alterations, including reduction in B lymphocytes and CD4 (+) T-cells, the increase in CD8 (+) T-cells, and expansion of natural killer (NK) cells result in increased susceptibility to infections and malignancies in this group of patients [17] [18]. Recent studies demonstrated that approximately $60 \%-90 \%$ of patients with psoriasis have pruritus [19] [20]. This value may change depending on the country, region, or race. In addition, the severity of itch in psoriatic patients has been thought to be no higher than that of atopic dermatitis; however, a recent meta-analysis of data from 22 clinical trials revealed no significant differences in the baseline severity of itch between these diseases [21]. In our case report, the pruritus was severe, causing sleep disturbance and difficulty to treat with antihistamines alone, but improved after resolved lesions. Regarding hyperpigmentation, psoriatic plaques in our case have a characteristic presentation of expanded projections of post-inflammatory hyperpigmentation surrounding the lesions and persist after improvement. Post-inflammatory hyperpigmentation is reported to be mediated by Tumor necrosis factor $\alpha$ and interleukin 17, and spots fade away over time once the flare is over [22] [23] differs from Woronoff pale annular zone that was known to appear around healing psoriasis lesions and was thought to be caused by a spastic vessel contraction or hypopigmentation [24]. Regarding treatments, the patient was followed over 2 years at the clinic and full assessment and investigations regularly were obtained to ensure patient safety before changing biological medications. To summarize clinical characteristics of this new variant as follow: 1) Early age of onset; 2) Mostly in Down syndrome cases; 3) PSEK-like in early 
presentation; 4) severely pruritic; 5) Perioral erythema with scales; 6) Fixed hyperkeratotic plaques over elbows, dorsal hands, knee joints, scrotum, and lower extremities accompanied with circinate scales in geographical distribution around and within plaques; 7) Expanded projection of post-inflammatory hyperpigmentation surrounding lesions; 8) Histopathology consistent with psoriasis; 9) Skin swab culture from the surface of pustules may be positive for pseudomonas infection; 10) Difficult to treat with conventional therapy alone. In conclusion, psoriasis can be presented with a new variant of a severely pruritic, recalcitrant type in Down syndrome cases which is clinically PSEK-like in presentation, but histopathologically consistent with psoriasis. However, early diagnosis and strict management are important in controlling the severity of the condition.

\section{Disclosure}

This study is an independent study and not funded by any of the drug companies.

\section{Conflicts of Interest}

The authors declare no conflicts of interest regarding the publication of this paper.

\section{References}

[1] Rachakonda, T.D., Schupp, C.W. and Armstrong, A.W. (2014) Psoriasis Prevalence among Adults in the United States. Journal of the American Academy of Dermatology, 70, 512-516. https://doi.org/10.1016/j.jaad.2013.11.013

[2] Parisi, R., Symmons, D.P.M., Griffiths, C.E.M. and Ashcroft, D.M. (2013) Global Epidemiology of Psoriasis: A Systematic Review of Incidence and Prevalence. Journal of Investigative Dermatology, 133, 377-385. https://doi.org/10.1038/jid.2012.339

[3] Rapp, S.R., Feldman, S.R., Exum, M.L., Fleischer, A.B. and Reboussin, D.M. (1999) Psoriasis Causes as Much Disability as Other Major Medical Diseases. Journal of the American Academy of Dermatology, 41, 401-407. https://doi.org/10.1016/S0190-9622(99)70112-X

[4] Wu, Y., Mills, D. and Bala, M. (2009) Impact of Psoriasis on Patients' Work and Productivity: A Retrospective, Matched Case-Control Analysis. American Journal of Clinical Dermatology, 10, 407-410. https://doi.org/10.2165/11310440-000000000-00000

[5] Kimball, A.B., Jacobson, C., Weiss, S., Vreeland, M.G. and Wu, Y. (2005) The Psychosocial Burden of Psoriasis. American Journal of Clinical Dermatology, 6, 383-392. https://doi.org/10.2165/00128071-200506060-00005

[6] Reich, K., Armstrong, A.W., Langley, R.G., Flavin, S., Randazzo, B., Li, S. and Blauvelt, A. (2019) Guselkumab versus Secukinumab for the Treatment of Moderate-to-Severe Psoriasis (ECLIPSE): Results from a Phase 3, Randomized Controlled Trial. The Lancet, 394, 831-839. https://doi.org/10.1016/S0140-6736(19)31773-8

[7] Langley, R.G.B., Krueger, G.G. and Griffiths, C.E.M. (2005) Psoriasis: Epidemiology, Clinical Features, and Quality of Life. Annals of the Rheumatic Diseases, 64 , 
ii18-ii23. https://doi.org/10.1136/ard.2004.033217

[8] Ladizinski, B., Lee, K.C., Wilmer, E., Alavi, A., Mistry, N. and Sibbald, R.G. (2013) A Review of the Clinical Variants and the Management of Psoriasis. Advances in Skin \& Wound Care, 26, 271-284. https://doi.org/10.1097/01.ASW.0000429778.10020.67

[9] Brandon, A., Mufti, A. and Gary Sibbald, R. (2019) Diagnosis and Management of Cutaneous Psoriasis: A Review. Advances in Skin \& Wound Care, 32, 58-69. https://doi.org/10.1097/01.ASW.0000550592.08674.43

[10] Darier, J. (1911) Progressive Symmetric Erythrokeratodermia Verrucosa. Bulletin de la Société Française de Dermatologie et de Syphiligraphie, 22, 252-264

[11] Gottron, H.A. (1923) Congenital Symmetrical Progressive Erythrokeratoderma. Archives of Dermatology, 7, 416.

[12] Khoo, B.P., Tay, Y.K. and Tan, S.H. (2000) Generalized Erythematous Plaques. Progressive Symmetric Erythrokeratoderma (PSEK) (Erythrokeratodermia Progressive Symmetrical). Archives of Dermatology, 136, 665-668.

[13] de Mello Guaraldi, B., Jaime, T.J., de Mello Guaraldi, R., Melo, D.F., Nogueira, O.M. and Rodrigues, N. (2013) Progressive Symmetrical Erythrokeratodermia-Case Report. Anais Brasileiros de Dermatologia, 88, 109-112. https://doi.org/10.1590/S0365-05962013000100016

[14] Raychaudhuri, S.P. and Gross, J. (2000) A Comparative Study of Pediatric-Onset Psoriasis with Adult-Onset Psoriasis. Pediatric Dermatology, 17, 174-178.

https://doi.org/10.1046/j.1525-1470.2000.01746.x

[15] Catarina, A., Resende, C., Vieira, A.P., Fernandes, J.C. and Brito, C. (2015) Plaque Psoriasis in a Patient with Down Syndrome-Potential Common Pathophysiology and Treatment Difficulty. Journal of the Portuguese Society of Dermatology and Venereology, 73, 147-150. https://doi.org/10.29021/spdv.73.1.357

[16] Marmon, S., De Souza, A. and Strober, B.E. (2012) Psoriasis and Down Syndrome: A Report of Three Cases and a Potential Pathophysiologic Link. Dermatology Online Journal, 18, 13. https://doi.org/10.5070/D305M5F4BX

[17] Ram, G. and Chinen, J. (2011) Infections and Immunodeficiency in Down Syndrome. Clinical \& Experimental Immunology, 164, 9-16.

https://doi.org/10.1111/j.1365-2249.2011.04335.x

[18] Murphy, M., Insoft, R.M., Pike-Nobile, L. and Epstein, L.B. (1995) A Hypothesis to Explain the Immune Defects in Down Syndrome. Progress in Clinical and Biological Research, 393, 147-167.

[19] Szepietowski, J.C. and Reich, A. (2016) Pruritus in Psoriasis: An Update. European Journal of Pain, 20, 41-46. https://doi.org/10.1002/ejp.768

[20] Amatya, B., Wennersten, G. and Nordlind, K. (2008) Patients' Perspective of Pruritus in Chronic Plaque Psoriasis: A Questionnaire-Based Study. Journal of the European Academy of Dermatology and Venereology, 22, 822-826. https://doi.org/10.1111/j.1468-3083.2008.02591.x

[21] Shahwan, K.T. and Kimball, A.B. (2017) Itch Intensity in Moderate-to-Severe Plaque Psoriasis versus Atopic Dermatitis: A Meta-Analysis. Journal of the American Academy of Dermatology, 76, 1198-1200. https://doi.org/10.1016/j.jaad.2017.02.002

[22] Wang, C.Q.F., Akalu, Y.T., Suarez-Farinas, M., et al. (2013) IL-17 and TNF Synergistically Modulate Cytokine Expression while Suppressing Melanogenesis: Potential Relevance to Psoriasis. Journal of Investigative Dermatology, 133, 2741-2752. https://doi.org/10.1038/jid.2013.237 
[23] Santa María, P., Valenzuela, F., Morales, C., De la Fuente, R. and Cullen, R. (2017) Lentiginous Eruption in Resolving Psoriasis Plaques during Treatment with Ixekizumab: A Case Report and Review of the Literature. Dermatology Reports, 9, Article ID: 7079. https://doi.org/10.4081/dr.2017.7079

[24] Woronoff, D.L. (1926) Die peripheren Veränderungen der Haut um die Effloreszenzen der Psoriasis vulgaris und Syphilis corymbosa. Dermatologische Wochenschrift, 82, 249-257. 